\title{
A NEW APPROACH ON CPS-BASED SCHEDULING AND WIP CONTROL IN PROCESS INDUSTRIES
}

\author{
Toshiya Kaihara \\ Kobe University \\ Rokkodai, Nada \\ 657-8501 Kobe, JAPAN
}

\author{
Yoshihiro Yao \\ Kobe University \\ Rokkodai, Nada \\ 657-8501 Kobe, JAPAN
}

\begin{abstract}
The cyber-physical system (CPS) concept is now attracting attention in systems engineering. We attempt to apply CPS to a fully automated factory management and control in processes such as semiconductor fabrication. We propose a novel structure, designated as a real-virtual integrated system, based on the CPS concept, and construct manufacturing scheduling and WIP control. It is designed to follow current dynamic changes and large future fluctuations at production sites simultaneously by executing dynamic scheduling of the real system and by simulation of the virtual system interactively. The effectiveness of the proposed scheduling method can be examined more accurately for possible adaptation to a shop floor.
\end{abstract}

\section{INTRODUCTION}

Cyber-physical systems (CPS) feature a tight combination of mutually coordinated computational and physical elements (Wolf 2007) (Lee 2008). CPS enables autonomous and flexible automation systems in several fields such as automotive systems, distributed robotics, and semiconductor fabrication systems.

Manufacturing companies are requested to manage widely various products in varying quantities within a short delivery lead time to respond to the increasing diversification of consumer needs and to strengthen global competitiveness. However, when producing small amounts and large varieties of products, many manufacturing shops are not operated efficiently because of the complexity of process flows and frequently changing circumstances related to operational delay, machine breakdown, and other problems, especially in automated semiconductor fabrications, because of the lack of flexibility. It is difficult to apply existing scheduling systems to such automated factories. Therefore, shop floor managers must control the shop based on their experiences and technical intuition with taking a long time, even in process industries, especially in complicated semiconductor fabrications.

In this paper, we propose a Real-Virtual Integrated Scheduling System (RVISS), which executes actual control of the real system and simulation of a virtual system interactively based on the CPS concept for these dynamically changing manufacturing environments. As described herein, RVISS responds to fluctuation of the real system using dynamic scheduling, and feeds back the fluctuation to the environment of the virtual system. Under this virtual environment, simulations are executed and future fluctuations are forecasted. Based on simulation results, scheduling parameters and scheduling methods are modified to respond to the future situations and improve long-term optimality. This paper reports the developed elemental methods and the proposed method's validity using computational experiments for realization of RVISS.

Auction-based scheduling was developed as a dynamic scheduling method intended for use with real systems. This method executes real-time scheduling using an auction protocol that imitates human contract negotiation. Therefore, it can decide work processes that must occur along with frequent situation changes. Results show that the delivery delay and the setup time can be greatly improved. Additionally, 


\section{Kaihara and Yao}

an integrated execution mechanism of scheduler and simulator was invented. Using this mechanism, simulations can be executed as needed, reflecting only those changing conditions of the real system to predict future situations. Finally, to evaluate the RVISS effectiveness, an experimental environment is established using a model plant and its controller. Actual hardware and schedulers can be connected in this environment, and the effectiveness of the proposed scheduling method can be examined more accurately for possible adaptation to real equipment.

\section{RVISS FOR A DYNAMICALLY CHANGING MANUFACTURING ENVIRONMENT}

Under a frequently changing manufacturing environment, dynamic scheduling is a suitable method to solve the problem only for the current short-term situation. It is repeated when the situation changes. Real-time scheduling using priority rules is a basic dynamic scheduling method. Some more effective methods have already been reported, such as periodically optimizing scheduling using the mixture of GA and priority rule (Eguchi 2005), but reactive scheduling executes re-scheduling when the gap separating the planned schedule and actual results increases (Tanimizu 2003).

Earlier studies have optimized logic and parameters under certain known dynamic conditions, and have confirmed their effectiveness using off-line simulation. Therefore, against the variation of dynamic condition itself such as demand fluctuation, there is a need to reconfigure the parameter and reconfirm it by executing off-line simulations.

This study aims at the realization of a scheduling system that can follow the variation of dynamic conditions themselves by executing the simulation every time as needed under the virtual system, which reflects the dynamic conditions of the real system.

Figure 1 presents the RVISS structure proposed for this study. The real system is controlled in response to the dynamically changing situation using a dynamic scheduling method such as real-time scheduling or reactive scheduling. Factors of fluctuation are the internal uncertainty component such as variation of processing time, and external fluctuation such as the increase and decrease in demand. Both factors are observed during the control process of the real system. They are fed back to the virtual system on the computer.

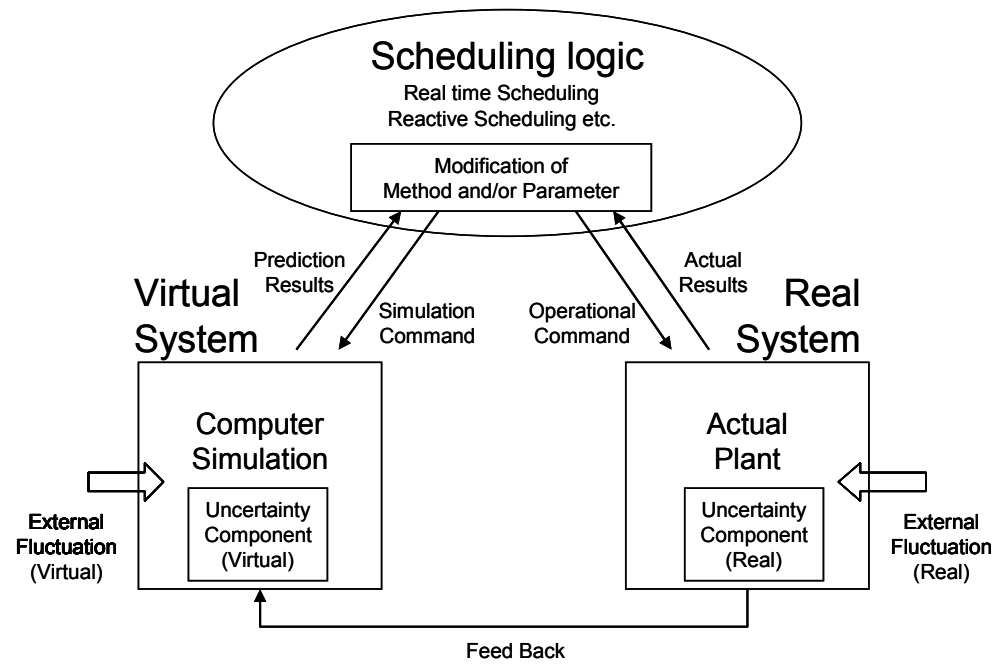

Figure 1: Structure of RVISS

For the virtual system, simulation is executed in real-time as needed using identical methods to control the real system. The timing and frequency of the simulation are determined according to the magnitude of the fluctuation of each system. With the results of the simulation, future conditions such as delivery delays or facility utilization are predicted. For cases in which problems are expected, scheduling 
parameters or methods are modified to prevent them. Using updated scheduling logic, control of the real system is continued.

In this way, RVISS is designed to follow current dynamic changes and large fluctuations in the future simultaneously by executing control of the real system and simulation of the virtual system interactively.

\section{AUCTION-BASED REAL-TIME SCHEDULING METHOD FOR A REAL SYSTEM}

For the real system in which processing conditions change frequently and for which processing does not necessarily proceed according to the initial schedule, expending long computational time to derive an optimal solution is not a suitable scheduling method. Instead, it is better to obtain a "semi-best" solution matching the current short-term situation which can be derived in a short time, and to repeat it when the situation changes (Fujii 2000). In this study, auction-based real-time scheduling is applied. This method, which imitates the contract negotiation of human society, is probably suitable for scheduling under frequently changing situations because it has the flexibility of being applicable to many cases by configuring the selection of items, bidders, and bids, and its algorithm has features of simplicity and speed in selecting the most suitable winner on the current status (Yao 2007, 2008) (Jayaola 2008) (Kaihara 2009).

In this case, the manufacturing equipment is defined as an item, the work piece as a bidder, and the slack time to the delivery date, the setup time, and the amount of rest process as the bid to execute auction-based scheduling. Figure 2 presents the flow of auction-based scheduling.

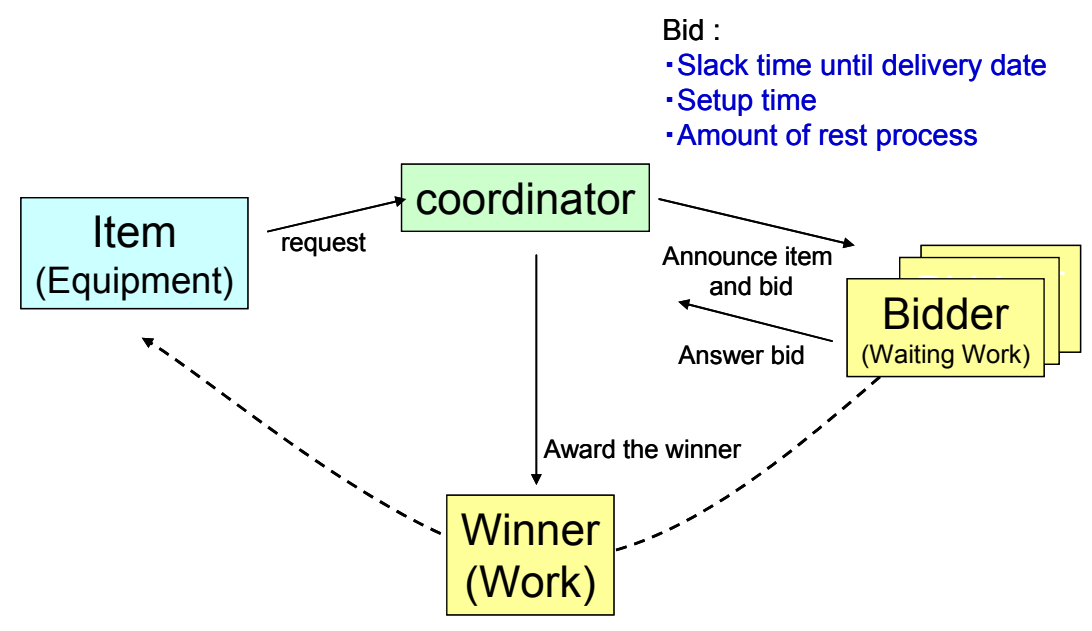

Figure 2: Auction based scheduling method

When one process has been finished and the machining equipment becomes empty, the auction described below is held to decide the next-processed work piece in a real-time manner.

- Item on auction: Equipment

- Bidder: Work piece

- Bid: Slack time to deliver date, setup time, and amount of rest processes are evaluated using the calculation formula and bid order shown in Table 1.

Flow of the auction:

Step 1: Coordinator announces the information of the item to the bidders

Step 2: Bidders return their bid prices to the coordinator

Step 3: If multiple bidders return the highest bid price:

a) If the next order of the bid in Table 1 exists, then go to step 1 with participants narrowed down to bidders with the highest bid price, or 


\section{Kaihara and Yao}

b) If there are no more orders of bid, then choose randomly from bidders with the highest bid price

Step 4: Award is given to the bidder with the winning bid

Table 1: Bid in auction for real-time scheduling

\begin{tabular}{|l|l|}
\hline Bid Order & \multicolumn{1}{|c|}{ Bid Calculation Formula } \\
\hline Bid 1 & $\begin{array}{l}\text { Setup time S=0 } \\
\text { or Slack time until delivery date } \mathrm{D}<\text { Highest limit } \mathrm{DH}_{\lim }: 1 \\
\text { Else : Quit }\end{array}$ \\
\hline Bid 2 & $\begin{array}{l}\mathrm{D}<\text { Lowest limit } \mathrm{DL}_{\lim }:-\mathrm{D} \\
\text { Else : Quit ( IF no bidder then go to Bid 3) }\end{array}$ \\
\hline Bid 3 & S \\
\hline Bid 4 & D \\
\hline Bid 5 & Amount of rest process $P$ \\
\hline
\end{tabular}

In this method, the setup time is assigned higher priority as evaluated bid than the delivery date (Bid 3 and 4 in Table 1). Consequently, the work pieces which have the same setup conditions are selected continuously, and the setup time is reduced. Additionally Bid 1 is proposed for increasing the lot size and for further decreasing the setup time. Parameter $\mathrm{DH}_{\text {lim }}\left(\mathrm{DL}_{\mathrm{lim}}\right)$ is the highest (lowest) limitation of the slack time to the delivery date. The work which needs setup time to the next processing and which has the slack time to deliver higher than $\mathrm{DH}_{\text {lim }}$ cannot participate in the auction. This bid order engenders increased process-waiting work piece, and consequently generates a larger lot size. By adjusting parameter $\mathrm{DH}_{\text {lim }}$, the delay of delivery date and setup time can be balanced appropriately.

Bid 2 in Table 1 has been introduced to eliminate the delivery delay in the case of arrival of work pieces which have a short slack time until the delivery date because of the emergency product order or processing delay of the previous process. The work which has the slack time lower than the parameter $\mathrm{DL}_{\text {lim }}$ is selected by priority in this auction step. The parameter $\mathrm{DL}_{\mathrm{lim}}$ also has the appropriate value to balance the delivery delay and the setup time.

\section{INTEGRATION OF REAL-TIME SCHEDULING AND SIMULATION}

In an existing manufacturing system, functions used in a manufacturing execution phase such as real-time scheduling and functions used in a planning phase such as simulation have been implemented as completely separate systems. They have been coordinated by exchanging necessary information through a database or inter-process communication. Therefore, if the configuration of the objective shop is updated, then it is necessary to modify both systems. Execution time takes longer to convert and transfer data between systems when they need to cooperate. However, in the proposed system, cooperation must be done rapidly because the real-time scheduling and the simulation must be executed concurrently. In this study, therefore the control mechanism has been developed by integrating the two systems, which can execute real-time scheduling and simulation using the same system models and processing algorithms.

Figure 3 presents the basic structure and the cooperating procedure of the integrated system. This system consists of two modules having real-time scheduling function and simulation function, but Cell object for equipment and the Coordinator object with auction algorithm are the common part in two functions. Entities of Work are implemented for each function using the same structured object.

When the simulation begins while executing real-time scheduling, Work objects for the real-time scheduling which is in Execution Waiting Queue and Cell Queue are copied and Work objects for the simulation are created. Then they set estimated finishing time for themselves and go into Execution Queue for the simulation. Works in the Auction Waiting Queue are copied and are put directly into the Auction Waiting Queue for the simulation. Results show that the simulation can be started immediately from just the moment and the status of executing real-time processing. 


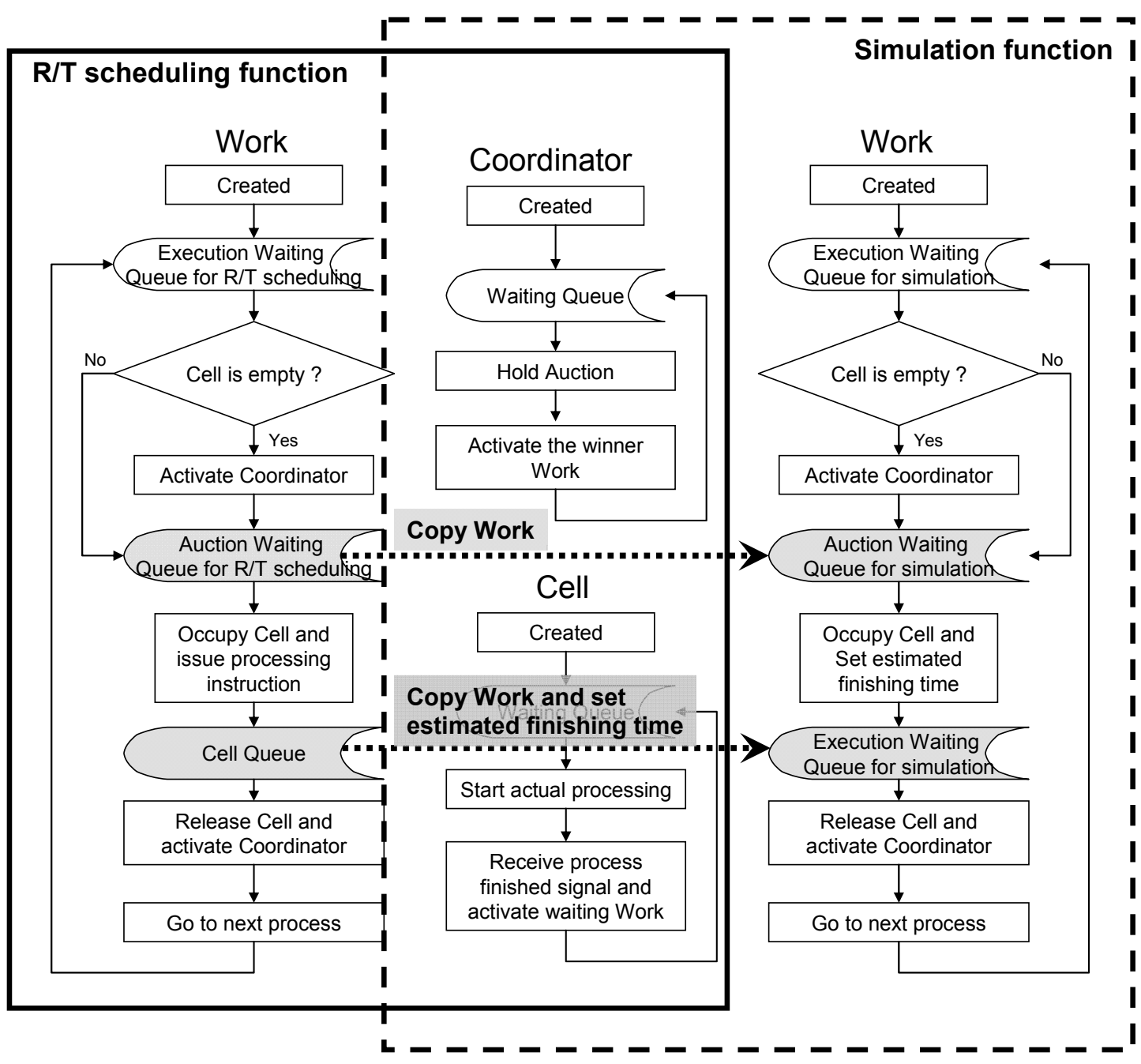

Figure 3: Integration of real-time scheduling and simulation system

\section{EXPERIMENTAL ENVIRONMENT FOR REAL-TIME SCHEDULING METHOD}

During the operating phase of the real-time scheduler, operational commands to the actual plant are decided according to the predefined scheduling logic under the condition of the operational result and the changing situation of the plant (Figure 4(c)). Generally, it is impossible to use actual manufacturing equipment to evaluate the effectiveness of this scheduling logic. Instead, a computational experimental environment such as a software emulator is usually built (Figure 4(a)). This software generates the changing condition of the manufacturing equipment virtually, and the reaction of the real-time scheduler is observed and its effectiveness is evaluated. In this environment, the experiment must be performed under the limited situation assumed by the researcher. Therefore, it is quite difficult to emulate all dynamic changes in the manufacturing equipment. This difficulty often causes unexpected problems during the phase of practical use of the scheduler.

To solve this problem, the experimental model plant (Figure 4 (b)) is probably effective to describe real dynamic situations including unexpected events. Some prior studies confirmed the effectiveness of model plant as a test bed for the development of a manufacturing system controller (Ramamoorthy 2007)(Agre 1995). 


\section{Kaihara and Yao}

In this study, the model plant presented in Figure 5 and Figure 6 is produced as an experimental environment of the proposed scheduling method. This plant consists of four milling machines: input, output, and buffer stations that are connected by a conveyer. Raw materials and finished products are stored in a warehouse.

Figure 7 presents control mechanisms of the model plant. Operational commands from the scheduler are sent to an equipment model controller via a command table. The controller drives actual equipment and its results are sent back to the scheduler via an event table. This mechanism is readily applicable to actual plants by replacing the model controller with one for actual equipment.

The proposed real-time scheduler is connected to this model plant and is confirmed to operate interactively. Using this experimental environment, the proposed scheduling method can be examined more accurately to ascertain whether it can respond effectively to situations that arise with actual equipment.

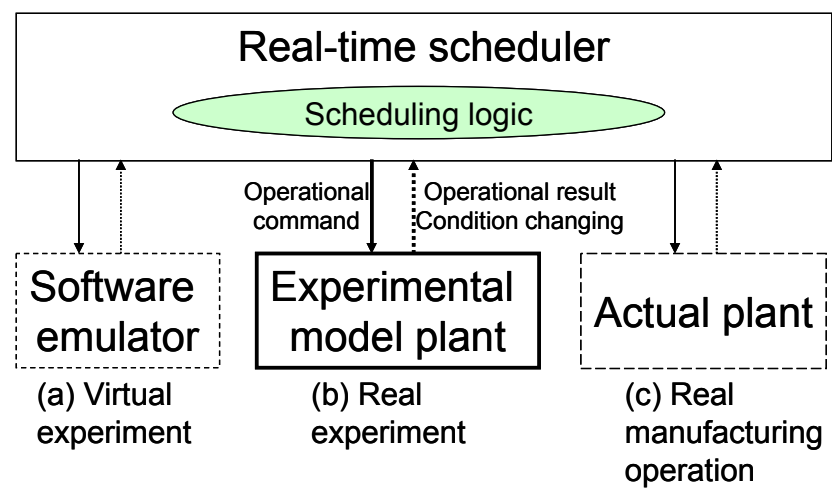

Figure 4: Method of experiment for real-time scheduler

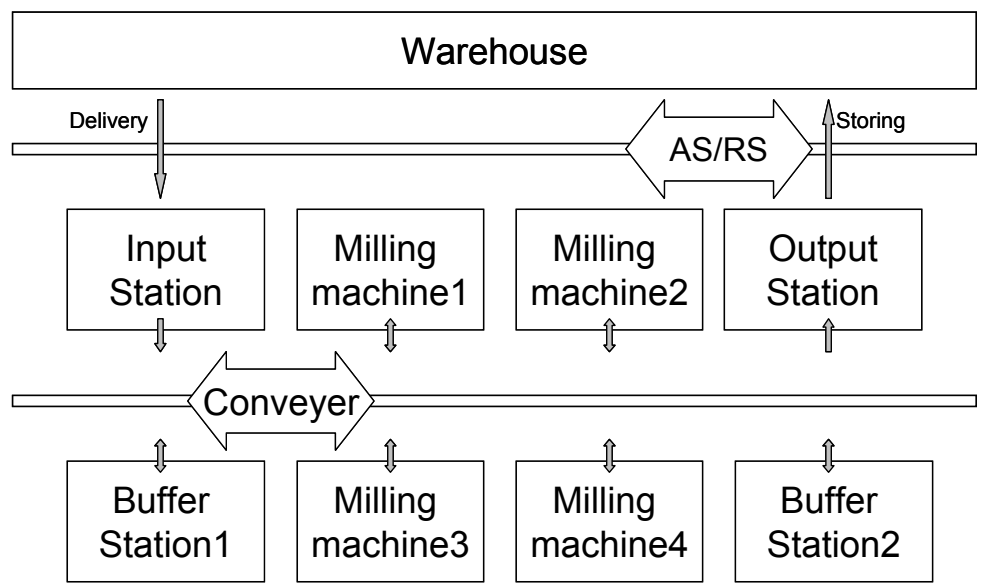

Figure 5: Experimental model plant 


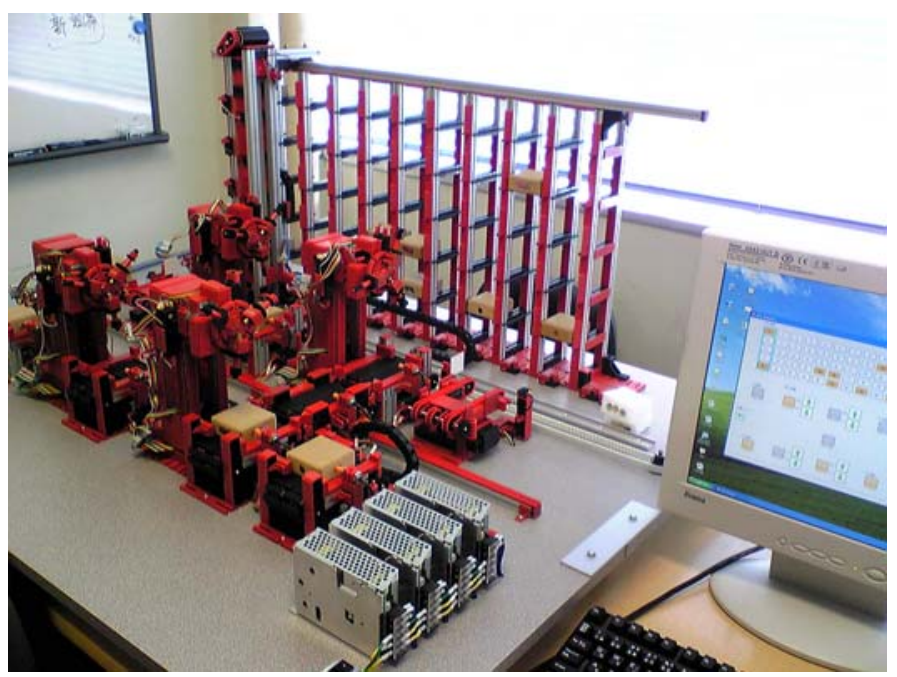

Figure 6: Overview of the model plant

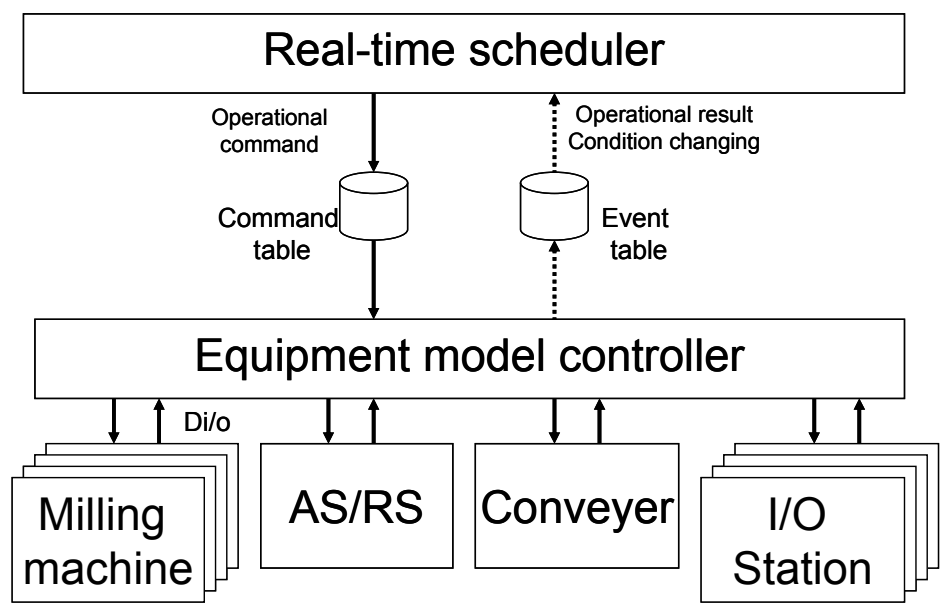

Figure 7: Structure of the experimental model plant

\subsection{Method of Executing the Simulation}

The method of executing the simulation is described below, followed by the method for modifying the scheduling precondition.

1) Execution timing

Select one timing below according to the amount of products, processing cycle time and/or frequency of the situation change.

- For every execution of the real-time scheduling at the time of finishing process or raw material arrival 


\section{Kaihara and Yao}

- Constant frequency, such as every $10 \mathrm{~min}$

- By occurrence of an event which has an impact on the current schedule, such as acceptance of the emergency product order and machine breakdown.

2) Simulation period

Sufficient length is necessary to detect the situation change of the shop. However, it is necessary to conduct simulations frequently and quickly to correspond to the dynamic situation flexibly. Therefore, the limitation exists by which the simulation period is constrained by the execution time. The appropriate simulation period also depends on the execution timing described above.

3) Evaluation of the result

Occurrence of the delay of delivery date, which increases the setup time and/or the amount of the raw material and in-process inventory, must be evaluated.

4) Scheduling precondition modification

Measures against the problem detected as a result of the simulation are the following.

- By increasing and decreasing the operating time of the shop and the number of workers, the shop capacity is adjusted to eliminate the delivery delay and decrease the in-process inventory. Shortterm correspondence is possible.

- Extending the material procurement lead-time or increasing the procurement lot size enlarges the production lot size and decreases the setup time. A reversal can decrease the in-process inventory. However, these measures require an amount of time that is dependent on the supplier.

- Expand the variety of products which one machine can process by preparing tools and jigs. The capacity of the shop and flexibility to the variety of product can be improved. However, it takes a long time and entails considerable cost.

One or more measures are selected depending on the features of the shop and are used as configurable parameters of this simulation system.

\subsection{Experiment of the Scheduling Precondition Modification}

An experiment was conducted to confirm the effectiveness of the scheduling parameter modification according to results of the simulation.

In this experiment, processing load on the shop changes during the execution of scheduling. The simulation detects that change in advance, increases the operating time of the shop to eliminate the delivery delay. Simulation parameters used in this experiment are below.

1) Timing of execution: For every execution of the real-time scheduling

2) Simulation period: Three days

3) Evaluation of the result: Average slack time to the delivery date of the finished work pieces

during the simulation period

4) Changing the scheduling precondition: Increasing the operating time of the shop

The shop is simplified to have one machine and products of three kinds to investigate the effect of the simulation clearly. The usual operating time is assumed as $16 \mathrm{hr}$ per day and the processing load is $15 \mathrm{hr}$, while only five days from the sixth day have a load of $21 \mathrm{hr}$.

First, Figure 8 presents results of execution of the real-time scheduling and the simulation concurrently without measures against the increase of the load. Slack time in the chart shows the actual result of the operation. The average slack time is the result of the simulation, which shows the prediction of the next three days. In this chart, slack time starts to decrease from the seventh day and the delivery delay occurs from the tenth day. However, average slack time begins to decrease at the fourth day, i.e., the simulation predicts the occurrence of the delivery delay. The following countermeasure against the increasing load has been taken.

- When the average slack time becomes lower than the threshold value $960 \mathrm{~min}$, the operating time of the next day is extended as $180 \mathrm{~min}$. 


\section{Kaihara and Yao}

Figure 9 shows the result of the scheduling. In this case, the operating time from seventh day to the twelfth day has been extended automatically by the simulation result, and the delivery delay has been eliminated.

The existing scheduling method can control the operating time against the previously known load change, but an unexpected load caused by an emergency product order or delay of processes must be managed by the human operator. However, as described above, the proposed method, which executes the simulation along with the real-time scheduling can predict the load change dynamically and control the operating time automatically and effectively.

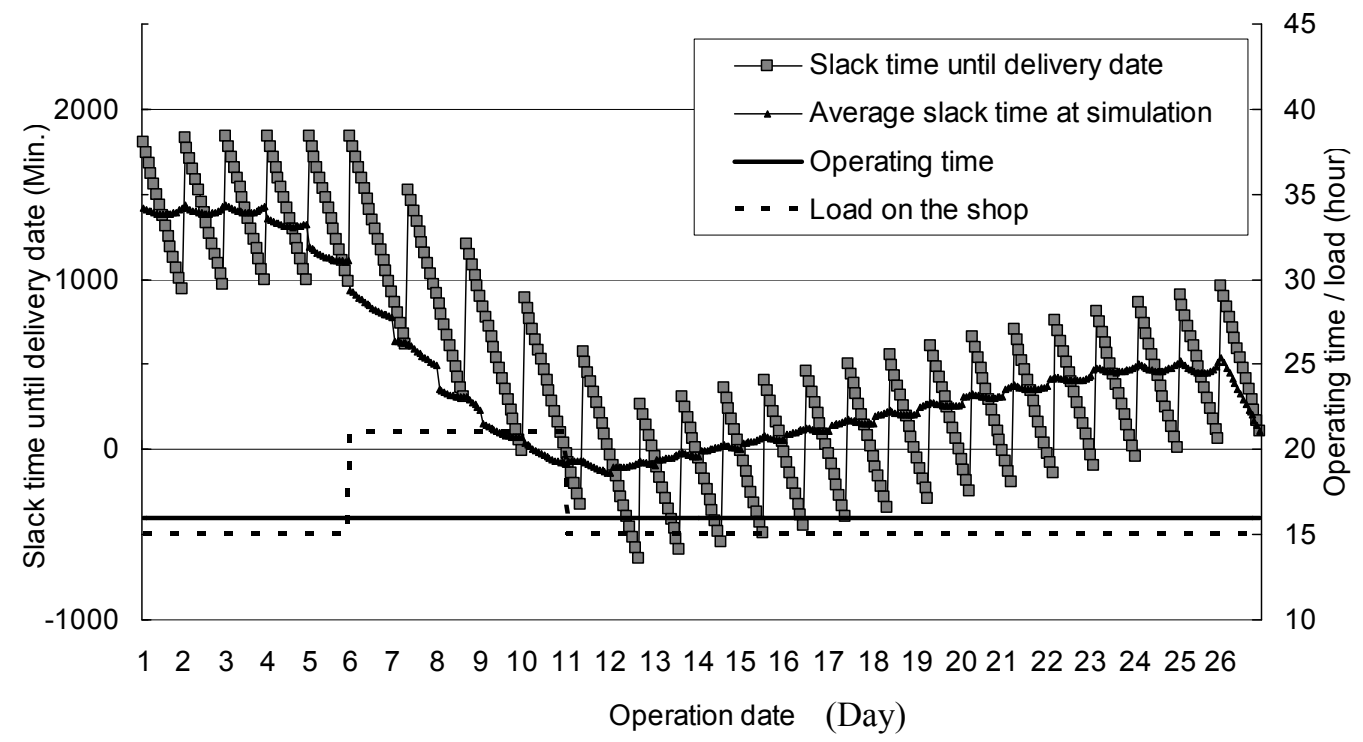

Figure 8: Scheduling result without operating time control

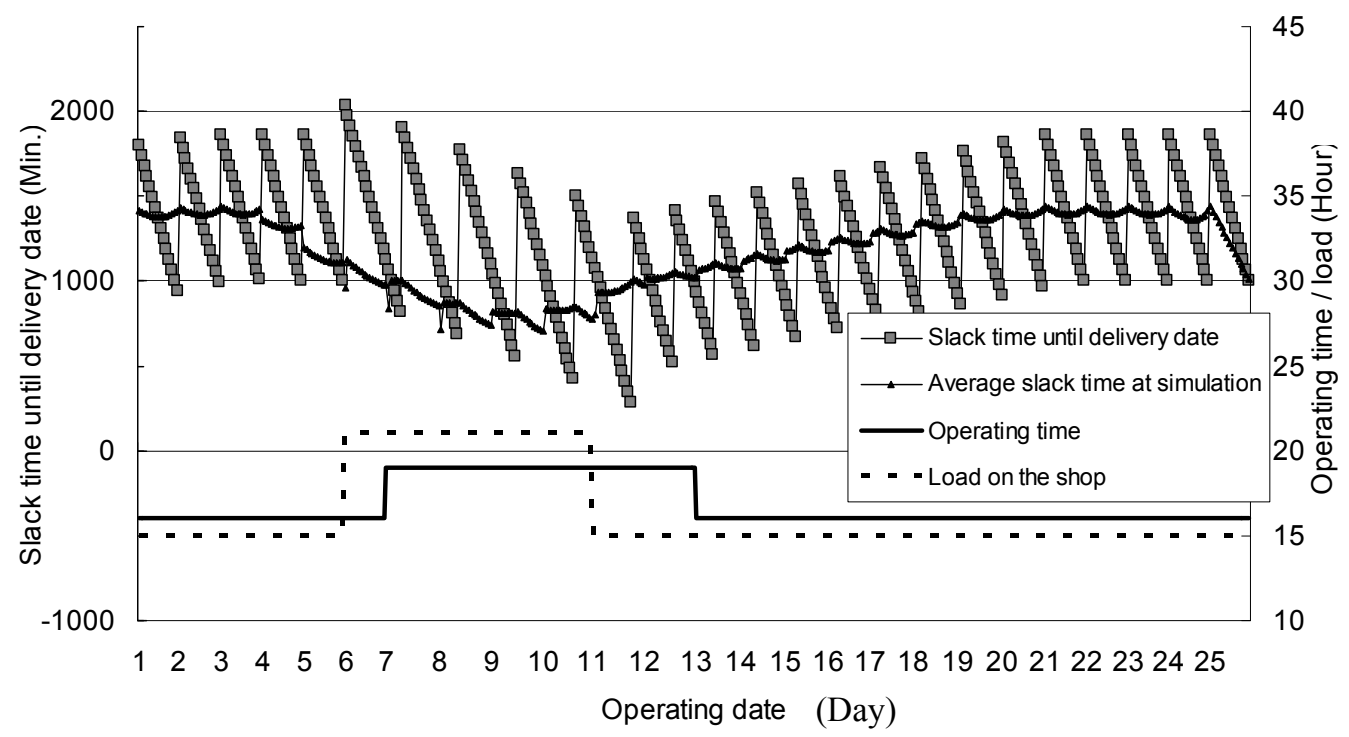

Figure 9: Scheduling result with operating time control 


\section{Kaihara and Yao}

\section{CONCLUSIONS}

In this study, the Real-Virtual Integrated Scheduling System (RVISS), which executes actual control of the real system and simulation of the virtual system interactively based on CPS concept was assessed for dynamically changing manufacturing environments. It responds to fluctuation of the real system using dynamic scheduling. A simulation was executed on the virtual system, which reflects the conditions of the real system and future fluctuation is forecasted. With this simulation result, scheduling parameters or scheduling methods are modified to improve long-term optimality. As a dynamic scheduling method used for a real system, auction-based scheduling has been proposed. Additionally integrated execution mechanism of a scheduler and simulator has been invented to execute simulation every time as needed, reflecting just the changing conditions of the real system. Finally, to evaluate the scheduling method, an experimental environment was established using the model plant and its controller, and its effectiveness was clarified using simulation experiments. The effectiveness of the proposed scheduling method can be examined more accurately for possible adaptation to processes such as semiconductor fabrication.

\section{REFERENCES}

Agre, J., Clare, L, Lee, J., Brandin, B., Hoskins, J., and Perrone, M.. 1995. "Autoconfigurable distributed control systems," Proceedings of the Second International Symposium on Autonomous Decentralized Systems (ISADS'95).

Eguchi, T., Oba F., and Kozaki S. 2005. "Dynamic scheduling using the mixture of a genetic algorithm and a priority rule," Transactions of the Japan Society of Mechanical Engineers, Series C, Vol.71, No.703, pp.1047-1053.

Fujii, S., Morita, H. and Tanaka, T., 2000, "A Basic Study on Autonomous Characterization of Square Array Machining Cells for Agile Manufacturing," Proc. 2000 Winter Simulation Conference, Vol. II, pp. 1282-1289.

Jayeola F. O. and Kaihara, T. 2008. "Value delivery in convergent supply network with mediating auctioneers", Proceedings of SICE Annual Conference 2008, 3B19-6, pp.3483-3486.

Kaihara, T., Fujii, N., Toide, S., and Ishibashi, H. 2009. "A study on optimization method for production scheduling with batch process by combinatorial auction", Proc. of International Symposium on Scheduling 2009, pp.266-267.

Lee, E. 2008. "Cyber Physical Systems: Design Challenges", University of California, Berkeley Technical Report, No. UCB/EECS-2008-8.

Ramamoorthy, K. 2007. "National instruments programmable automation controller for reconfigurable logic control: Implementation and evaluation," Engineering Research Center for Reconfigurable Manufacturing Systems ERC/RMS, University of Michigan, September 2007.

Tanimizu, Y., Sakaguchi, T., and Sugimura, N. 2003. "Genetic Algorithm Based Reactive Scheduling : 1st Report, Modification of Production Schedule for Delays of Manufacturing Processes," Transactions of the Japan Society of Mechanical Engineers, Series C, Vol.69, No.685, pp.2458-2463.

Wolf, W. 2007. "The Good News and the Bad News : Embedded Computing Column," IEEE Computer, Vol.40, No.11. p.104.

Yao, Y., Kaihara, T., Sashio, K. and Fujii, S. 2007. "A Study on Automated Scheduling Methodology for Machining Job Shop," Proc. 12th IEEE International Conference on Emerging Technologies and Factory Automation, CD-ROM.

Yao, Y., Kaihara, T., Sashio, K., Fujii, S. and Morikawa K. 2007. "A scheduling methodology for dynamically changing manufacturing environment”, Proc. of 2008 International Symposium on Flexible Automation, CD-ROM. 


\section{AUTHOR BIOGRAPHIES}

TOSHIYA KAIHARA is a Professor of Graduate School of System Informatics at Kobe University, Kobe, Japan. He received the B.E. and M.E. degrees in precision engineering from Kyoto University, Kyoto, Japan, and Ph.D. degree in mechanical engineering from Imperial College, University of London, London, UK. His research interests include a theory of sociological multi-agent and its application into manufacturing system, logistics system and social system. He is a member of CIRP, IFIP, IEEE, SICE, IEEJ, JSME, and many other academic societies.

YOSHIHIRO YAO was employed at Komatsu Ltd. in 1985 and worked as a chief researcher at Manufacturing Engineering Research Center and as a project manager of manufacturing system at Osaka Plant until 2006. He then established i-Process Planning LLC and started his business as a manufacturing system consultant for operational efficiency improvement. Concurrently he studied as a student at the Graduate School of Science and Technology at Kobe University and received his Ph.D. for his work on manufacturing scheduling and simulation in 2012. 